\title{
Translating medical science around the world
}

I mproving the translation of basic research into the clinic is fast becoming a priority not just in the US but also in Europe.

In the US, one of the biggest initiatives to enhance translational research is sponsored by the NIH through its Clinical and Translational Science Awards (CTSAs). The first CTSAs were awarded to 12 institutions in October last year, a second series of recipients was, at press time, set to be announced in September this year, and applications for next year's awards are soon to be evaluated. Funding is for a 5-year period, and a total of almost $\$ 100$ million was provided to the 12 institutions for the first year alone.

The goal of CTSAs is to eliminate barriers between clinical and basic research, in part by assembling interdisciplinary teams covering the spectrum of research (1). At the University of Pennsylvania (Penn), Garret FitzGerald - professor of medicine and pharmacology, director of Penn's Institute for Translational Medicine and Therapeutics (ITMAT), and principle investigator for Penn's CTSA - told the JCI that "the CTSA award has enabled ITMAT to broaden its focus and become a transinstitutional and trans-school enterprise." He says this is bringing together people from diverse disciplines, something he considers essential if academia is going to successfully reenter the drug discovery arena and safely translate basic science into therapeutics.

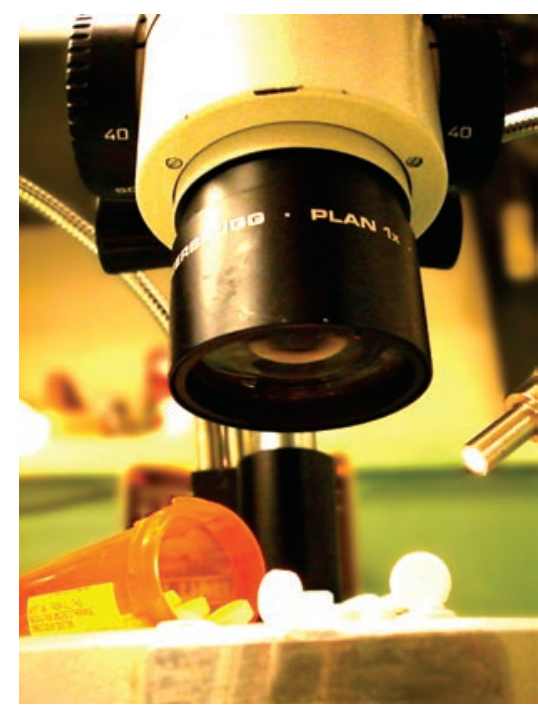

New initiatives to enhance the translation of basic research into improved patient care are being developed around the world.
FitzGerald is also in a position to assess the changes that are occurring in translational research on the other side of the Atlantic he served last year, for example, on the panel that advised the National Institute for Health Research (NIHR) in the United Kingdom on where to establish their comprehensive Biomedical Research Centres (BRCs) $(2,3)$, a venture that he considers "very healthy for UK biomedicine," and he is about to chair a review committee for the British Heart Foundation to establish interdisciplinary Centres of Excellence, again focused on translational enterprise. He told the JCI that these two approaches build on earlier Wellcome Trust initiatives to fund translational research and are an impressive step forward for biomedical research in the UK.

Each BRC is a partnership between a university and a National Health Service (NHS) trust - a regional unit of the publicly funded health care system in the UK. Funding was provided to five comprehensive BRCs (three sited in London, one in Cambridge, and one in Oxford), which will cover a wide range of areas, and six specialist BRCs, which will work in very defined areas such as cancer, ophthalmology, and mental health. Funding began in April of this year and will provide more than $£ 450$ million (approximately $\$ 900$ million) over the next five years.

One big difference between the money provided to researchers in the US through CTSAs and that provided to researchers in the UK at the BRCs is that in the UK, the money cannot be used for preclinical studies; it must all be used for patient-based clinical research. As Graham Lord, professor of medicine and deputy director of the comprehensive BRC at King's College London, noted to the JCI, "This provides a challenge to do something different." As one of the five comprehensive BRCs, the partnership between King's College and Guy's and St. Thomas' NHS Foundation Trust created a new Faculty of Translational Medicine to help them achieve the overall goal of the BRCs of improving patient care. Lord believes that creating the new faculty, which will be based on two floors of Guy's and St. Thomas' Hospital and colocated with new clinical research facilities, will provoke interactions among faculty members and others from different backgrounds (senior NHS clinicians, university academics, dentists, healthcare professionals, NHS managers, and patients) and that fostering such interactions is necessary to effect the cultural shift required to generate novel ideas and approaches to patient care.

Although the five comprehensive BRCs competed against each other for UK funding, one of the requirements of the recipients is that they engage with each other to compete with clusters of biomedical research facilities around the world, such as the cluster found in Boston. To help achieve this, the UK government is creating a Global Medical Excellence Cluster, which will foster interactions among the five centers that were awarded comprehensive BRC status as well as between these centers and biotechnology and pharmaceutical companies (4).

Other countries in Europe are also developing strategies to improve translational research (5). For example, the Center for Drug Research, Development, and Safety (ZAFES) in Germany was founded at the Johann Wolfgang Goethe University in Frankfurt am Main in 2002 as a center of excellence intended to more rapidly develop innovative drugs by combining insights from academia, the pharmaceutical industry, and biotechnology.

FitzGerald pointed out to the JCI that one of the current limitations is human capital and that attracting the best clinicians and scientists of the future might be difficult until the community comes up with a unifying, global tag for this type of research and training (6). Unfortunately, coming up with a name is only one of many challenges facing the translation of basic research into the clinic.

\section{Karen Honey}

1. NIH. 2006. NIH launches national consortium to transform clinical research. http://www.nih.gov/ news/pr/oct2006/ncrr-03.htm.

2. National Institute for Health Research. 2006. Biomedical Research Centres. http://www.nihr.ac.uk/ infrastructure_biomedical_research_centres.aspx.

3. UK Department of Health. 2006. New Health Research Centres of excellence announced. http:// www.gnn.gov.uk/Content/Detail.asp?ReleaseID =2 49162\&NewsAreaID $=2$.

4. Griffiths, K. 2006 December 14. Blair promotes 'UK medical centre of excellence'. The Daily Telegraph. http://www.telegraph.co.uk/money/main. jhtml?xml=/money/2006/12/14/cnmedica $14 . x m l$.

5. Pain, E. 2007. European programs offer translational training. Science. 317:968-969.

6. FitzGerald, G.A. 2007. Clinical pharmacology or translational medicine and therapeutics: reinvent or rebrand and expand? Clin. Pharmacol. Ther. 81:19-20. 